$39^{\text {th }}$ AIAA/ASME/SAE/ASEE Joint Propulsion Conference

Huntsville, AL 20-23 July 2003

\title{
Computational Modeling of Stationary Plasma Thrusters
}

\author{
Justin W. Koo* and Iain D. Boyd ${ }^{\dagger}$ \\ Department of Aerospace Engineering \\ University of Michigan \\ Ann Arbor, Michigan 48109
}

\begin{abstract}
A 2-D axisymmetric hybrid PIC-MCC model of the acceleration channel and near-field of dielectric wall-type Hall thrusters is tested on an SPT-100 type and a UM/AFRL P5 magnetic field configuration. The location of both the virtual cathode line (VCL) and virtual anode line (VAL) are varied on the SPT-100 type magnetic field configuration to study the model dependence on these parameters. A P5 simulation with and without doubly charged xenon is provided. For the particular SPT-100 type magnetic field configuration studied, there is a negligible dependence of performance parameters and plasma properties on the location of the VCL; however, the dependence on the location of the VAL is far more pronounced. Moving the VAL downstream results in decreased plasma density both in the acceleration channel and upstream of the VAL, diminished thruster performance parameters, and a lowering of the electron energy in the near-field region. Inclusion of $\mathrm{Xe}^{++}$led to little change in the performance characteristics of the thruster.
\end{abstract}

\subsection{Introduction}

Experimental study of Hall thrusters has far outpaced the computational study of these devices in the decades since their introduction; however, interest remains in the development of robust, accurate, and efficient Hall thruster codes. Among the numerous benefits of such a computational model would be the ability to perform full spacecraft integration studies, the means to quantify chamber effects in experimental tests, and the capacity to perform virtual life tests for a fraction of the cost of actual life tests.

Many new computational models of the plasma properties inside Hall thrusters have been developed recently. They range from 1-D and 2-D steady-state models such as those by Keidar et al. ${ }^{1}$, Ahedo et al. ${ }^{2}$, to 2-D fluid models by Roy and Pandey ${ }^{3}$, to full 1-D and 2-D time dependent models by Komurasaki and Arakawa $^{4}$, Fife ${ }^{5}$, Boeuf and Garrigues ${ }^{6}$, and, most recently, by Hagelaar et al. $^{7}$ and others. These computational models have reached a stage of refinement where, with a priori knowledge of a particular experimental flow condition, a reasonably representative computational solution can be achieved in a matter of hours.

Unfortunately, these solutions are by no means sufficiently reliable to prompt widespread use in the

\footnotetext{
*Graduate Student, Student Member AIAA, kooj@engin.umich.edu

†Professor, Department of Aerospace Engineering, Associate Fellow AIAA
}

Hall thruster design community. In particular, although computational models can be tuned to produce plasma structures and electric fields which are qualitatively similar to results obtained from internal experimental diagnostic studies, a single model guaranteeing fidelity over a wide range of operating conditions is not yet feasible. As part of our continued development of a robust Hall thruster model, this paper studies the dependence of a particular model on anode and cathode boundary conditions. In addition, it demonstrates the incorporation of $\mathrm{Xe}^{++}$into the computational model. The performance of the model is assessed through application to the SPT-100 and P5 Hall thrusters.

\subsection{Computational Model}

This model provides a 2-D axisymmetric hybrid PICMCC description of the acceleration channel and nearfield of dielectric wall-type Hall thrusters. It is based on a quasi-neutral plasma description where heavy particles $\left(\mathrm{Xe}, \mathrm{Xe}^{+}\right.$, and $\left.\mathrm{Xe}^{++}\right)$are treated with a PICMCC model. The electron fluid is modeled with a 1-D electron energy model. Plasma potential is calculated using a 1-D Ohm's Law formulation.

\subsection{Magnetic Field Configurations}

Two different magnetic field configurations are considered. The UM/AFRL P5 field configuration was obtained experimentally using a Hall probe. The SPT100 type field configuration is calculated using a Poisson solver $\left(\nabla^{2} B=0\right)$ with channel wall boundary conditions derived from experimental sources. For both thrusters, the magnetic field configuration is used to calculate the magnetic field streamfunction via the following relations: 


$$
\frac{\partial \lambda}{\partial r}=r B_{z} \quad \frac{\partial \lambda}{\partial z}=-r B_{r}
$$

The normal derivative with respect to field lines can be written as follows:

$$
\frac{\partial}{\partial \hat{n}}=r B \frac{\partial}{\partial \lambda}
$$

Equipotentials of the streamfunction correspond to magnetic field lines and are used to formulate the 1-D Ohm's Law and 1-D electron energy equations.

\subsection{1-D Ohm's Law Formulation}

A fundamental premise of the reduction of this 2-D problem into a 1-D Ohm's Law formulation is the idea that there is a constant defined for each individual magnetic field line which is a balance between the electrostatic pressure and the electron thermal pressure along field lines. This concept, first introduced by Morozov $^{8}$, is known as the thermalized potential and is defined as follows:

$$
\phi^{*}(\lambda)=\phi(\lambda)-\frac{k T_{e}}{e} \ln \left(\frac{n}{n^{*}}\right)
$$

where $n^{*}$ is an arbitrary constant. Thus, along a given field line, the potential and density vary such as to maintain a constant thermalized potential while from a given field line to a different field line, the value of the thermalized potential may vary.

The electrostatic field calculation is based on the assumption that there is no net buildup of charge throughout the domain. This requires the sum of the electron and ion currents to balance throughout the domain as follows:

$$
\begin{aligned}
I_{T} & =\int_{S} j_{e} \partial S+\int_{S} j_{i} \partial S \\
= & \int_{S} e n_{e} \mu_{e} r B\left(-\frac{\partial \phi^{*}}{\partial \lambda}-\left[\ln \left(\frac{n_{e}}{n^{*}}\right)-1\right] \frac{k}{e} \frac{\partial T_{e}}{\partial \lambda}\right) \partial S \\
& +\int_{S} e n_{i} u_{i} \partial S
\end{aligned}
$$

This equation is summed from the VAL to the VCL and a closed form solution for the total current can be derived, as shown in the next column. Typically, a line of constant thermalized potential near the domain exit is chosen as the VCL and an equivalent line near the injector is defined as the VAL. Once the total current is known, the derivative of the thermalized potential can be calculated directly and a full thermalized potential can be constructed. The potential is then calculated along field lines through the use of the thermalized potential and then extrapolated through the whole domain. The location of the VAL and VCL are varied in the present investigation to study the model dependence on these parameters.

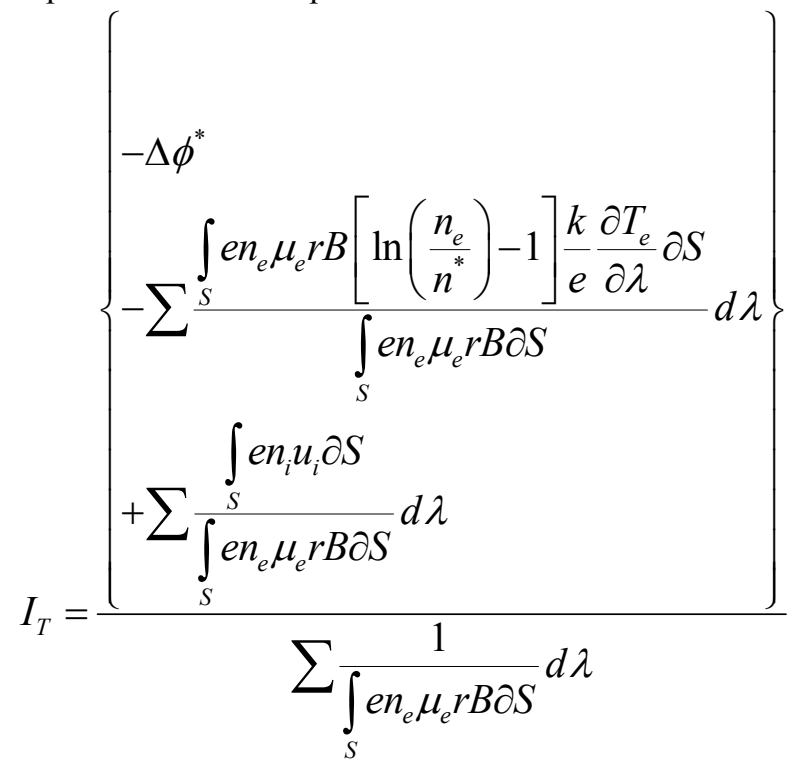

\subsubsection{Electron Mobility}

The transverse magnetic field electron mobility is needed for calculation of the electrostatic field. To ensure that the electron mobility does not drop catastrophically in regions of neutral depletion, the electron momentum transfer frequency is supplemented by an effective wall scattering term suggested by Boeuf and Garrigues ${ }^{6}$. This leads to the following term for the electron momentum transfer frequency:

$$
v_{\text {mom }}=v_{\text {neutrals }}+v_{\text {walls }}
$$

where,

$$
\begin{gathered}
v_{\text {neutrals }}=2.5 * 10^{-13} n_{a} \\
v_{\text {walls }}=\alpha * 10^{7}
\end{gathered}
$$

The value for $\alpha$ is consistent with the electron energy loss frequency presented later in this paper. This effective momentum transfer frequency is then used in the classical description of the transverse magnetic field electron mobility:

$$
\mu_{e}=\frac{e}{m v_{\text {mom }}} \frac{1}{1+\left(\frac{\omega_{e}}{v_{\text {mom }}}\right)^{2}}
$$

where $\omega_{\mathrm{e}}$ is the electron cyclotron frequency.

\section{$\underline{2.3 \text { Ionization Source Terms }}$}

Both singly charged and doubly charged xenon particles can be considered in this model. The general 
form of the source term for excitation of both charged species from the ground state is as follows:

$$
\frac{\partial n_{i}}{\partial t}=n_{p} n_{a} k_{\text {neutral }}^{i} \Delta t
$$

where the right hand side consists of the plasma density, neutral density, and ionization rate of the particular species, respectively. Newly created ions are created throughout the domain at each timestep based on average cell source rates.

Excitation of singly-charged xenon to doubly-charged xenon (referred to in this paper as stepwise ionization) is modeled with a separate Monte Carlo Collision (MCC) ionization model.

Ionization and energy loss rates are taken from Garrigues et al. ${ }^{9}$ These ionization rates are provided below in Figure 1.

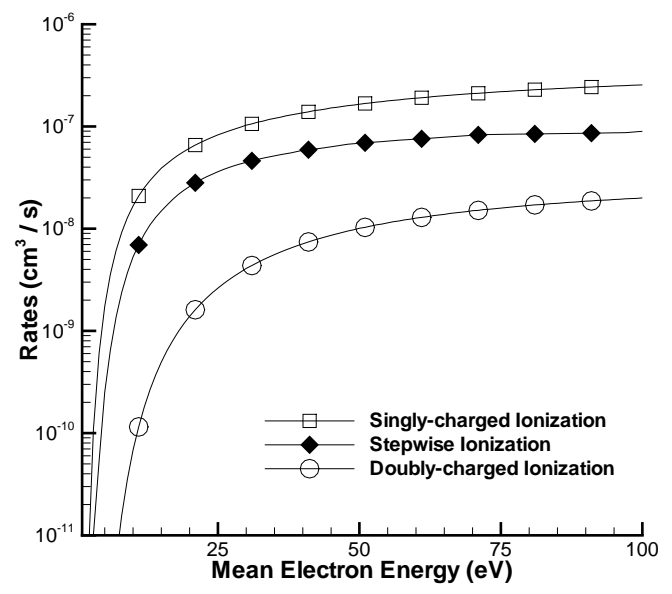

Figure 1. Ionization Rates

\section{$\underline{2.4 \text { Heavy Particle Behavior }}$}

The motion of the heavy particles is based on a first order advection scheme. New ion velocities are calculated from the electrostatic field equations at halfsteps relative to ion positions. Quantities are updated in the same manner as a classical leapfrog update scheme.

$$
\begin{aligned}
& u^{t+\frac{\Delta t}{2}}=u^{t-\frac{\Delta t}{2}}+\frac{e E}{m} \Delta t \\
& x^{t+\Delta t}=x^{t}+u^{t+\frac{\Delta t}{2}} \Delta t
\end{aligned}
$$

Neutrals are injected at the anode to match the desired mass flow rate. They are removed from the simulation via a Monte-Carlo Collision (MCC) model due to ionization. Newly ionized $\mathrm{Xe}^{+}$and $\mathrm{Xe}^{++}$particles have a Maxwellian velocity distribution based on a $1000 \mathrm{~K}$ reference temperature.

Wall recombination occurs when ions strike any thruster wall and results in the formation of an equal number of fully accommodated $(1000 \mathrm{~K})$ neutral particles. Neutral scattering at the wall is also based on full thermal accommodation.

\subsubsection{Monte Carlo Collision (MCC) Model}

To calculate both neutral depletion due to ground state ionization and singly charged xenon depletion due to stepwise ionization, an MCC model is used. First, a probability of collision, $\mathrm{P}_{\mathrm{C}}$ (generally $<<1$ ) is calculated as follows:

$$
\begin{aligned}
P_{C}^{\text {neutral }} & =n_{\text {plasma }} k_{\text {neutral }}^{i} \Delta t \\
P_{C}^{\text {stepwise }} & =n_{\text {plasma }} k_{X e^{+}}^{i} \Delta t
\end{aligned}
$$

where, $k_{\text {neutral }}^{i}$ and $k_{X e^{+}}^{i}$ are the neutral ionization and $\mathrm{Xe}^{+}$ionization rates, respectively. Next, at every timestep, each neutral and $\mathrm{Xe}^{+}$macroparticle is assigned a random number from 0 to 1 . If this random number is less than $\mathrm{P}_{\mathrm{C}}$, then a collision event is simulated and the particle type is removed (for neutral depletion) or changed (for stepwise ionization).

\section{$\underline{2.5 \text { Electron Energy }}$}

Electrons are assumed to be isothermal with a Maxwellian energy distribution along magnetic field lines. This allows for a 1-D decomposition of the electron energy equation across field lines. The complete electron energy equation is as follows:

$$
\begin{gathered}
\frac{\partial}{\partial t}\left(n_{e} \varepsilon\right)+\vec{\nabla} \bullet\left[\frac{5}{3} n_{e} \varepsilon \vec{u}_{e}-\frac{10}{9} n_{e} \mu_{e} \varepsilon \vec{\nabla} \varepsilon\right] \\
=-n_{e} \vec{u}_{e} \bullet \vec{E}-n_{e} \varepsilon v_{\text {loss }}(\varepsilon)
\end{gathered}
$$

where the electron energy loss frequency is defined as:

$$
v_{\text {loss }}(\varepsilon)=v_{e-n}\left(n_{a}, \varepsilon\right)+v_{\text {stepwise }}\left(n_{X e^{+}}, \varepsilon\right)+v_{\text {walls }}(\varepsilon)
$$

The first term composing the loss frequency is the frequency of electron energy losses associated with ionization and excitation from the ground state, the second term represents the electron energy losses associated with stepwise ionization of $\mathrm{Xe}^{+}$, and the third term is a wall-loss term. The form chosen for this wallloss term, as suggested by Boeuf and Garrigues ${ }^{6}$, is:

$$
v_{\text {walls }}(\varepsilon)=\alpha * 10^{7} \exp \left(\frac{-\beta}{\varepsilon}\right)
$$

For the P5 and SPT-type model results presented here, particular choices of $\alpha$ and $\beta, \alpha=0.11: \beta=30 \mathrm{eV}$ and $\alpha=0.09$ and $\beta=20 \mathrm{eV}$, respectively, are selected to 
provide representative results. The electron energy loss frequency is presented below in Figure 2.

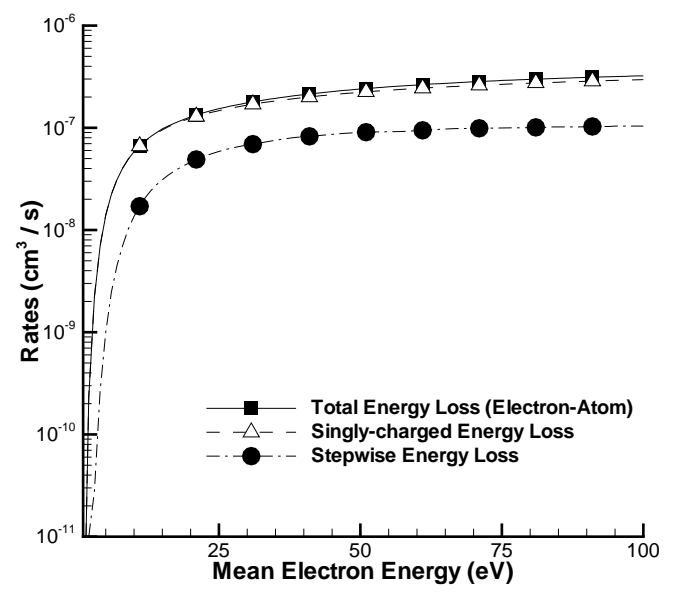

Figure 2. Electron Energy Loss Rates

The electron energy equation above can be recast in the form of an ordinary differential equation with a dependence on the electron energy alone. Volume integration of the electron energy equation is used to evaluate the coefficients in a smooth manner. Stable integration of the resulting ODE requires a timestep far smaller than the timestep used for heavy particle evolution. As suggested by Fife $^{5}$, the electron energy equation is subcycled 100 times for every single heavy particle timestep to ensure accurate integration.

\subsubsection{Electron Energy Boundary Conditions}

The active domain simulated by the electron energy equation is defined by the location of the VAL and VCL. Although the VAL and VCL locations for the electron energy equation need not be the same as for the potential calculation, for consistency, the same VAL is chosen for both the electron energy and potential calculation and, likewise, the same VCL is chosen for both the electron energy and potential calculation. The electron energy at the VAL is fixed at $3 \mathrm{eV}$ and at the VCL is fixed at $2 \mathrm{eV}$ for both the P5 and SPT-100 type configurations.

\section{$\underline{2.6 \text { Computational Details }}$}

The computational model is compiled with SUN $\mathrm{f} 77$ to run on a Sun Ultra $10 / 440 \mathrm{MHz}$ workstation. A simulation typically contains 60,000 ion macroparticles and 200,000 neutral macroparticles. The heavy particle timestep is limited to the time needed for a perfectly accelerated particle to cross half a computional cell which results in a timestep of about $2.5 \times 10^{-8}$ seconds. A typical solution time is 24 hours.

\subsection{Results and Discussion}

3.1 Cathode Study

\subsubsection{Motivation}

For some thrusters, a significant fraction of the ion acceleration can be observed over $10 \mathrm{~cm}$ from the exit plane of the thruster. ${ }^{10}$ Also, in earlier simulations of this model, evaluating performance across vertical planes at different axial locations from the thruster led to different performance results. This behavior indicates that the size of the model domain has an impact on the performance of this model.

\subsubsection{Configuration}

The location of the VCL is varied to study its effect on the performance of this model on the SPT-100 type magnetic field configuration. For this study, the three VCLs chosen correspond to different lines of constant thermalized potential (third, fifth, and eleventh line from the domain exit) for the same magnetic field configuration. The VAL location chosen is the third line of constant thermalized potential. The three configurations are shown in Fig. 3.

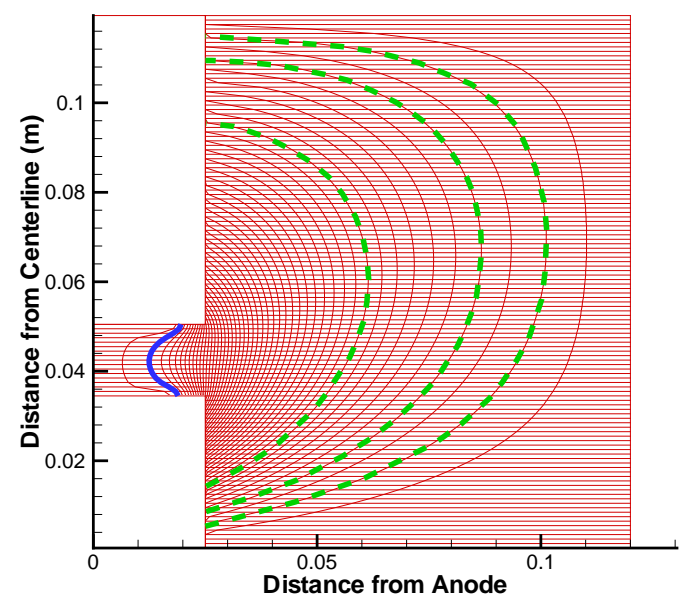

Figure 3. VAL in bold; Dashed lines are VCL (from left to right) $\lambda=(11), \lambda=(5), \lambda=(3)$

Ionization is permitted upstream of the VAL, but the centerline electron energy and thermalized potential are enforced as constants upstream of the VAL. To reduce the influence of the "far" near field domain on the area of active solution of the electron energy and potential, ionization is prohibited downstream of the VCL. For simplicity, the outer walls of the thruster are assumed to be made of dielectric material. Consequently, wall recombination occurs on the outer walls of the thruster. 
Heavy particles continue to be tracked to the domain exit boundaries where performance data is evaluated.

All three models are run with a xenon mass flow rate of $5 \mathrm{mg} / \mathrm{s}$ and imposed potential drop of $275 \mathrm{~V}$ between the VAL and VCL. Doubly charged xenon is not considered in this study.

\subsubsection{Mean Plasma Density}

Figures 4-6 show the mean plasma density for this study. With the exception of a small region of the nearfield domain near $\mathrm{x}=0.09 \mathrm{~m}$ and $\mathrm{r}=0.02 \mathrm{~m}$, the location and density of plasma is virtually identical for all three cases.

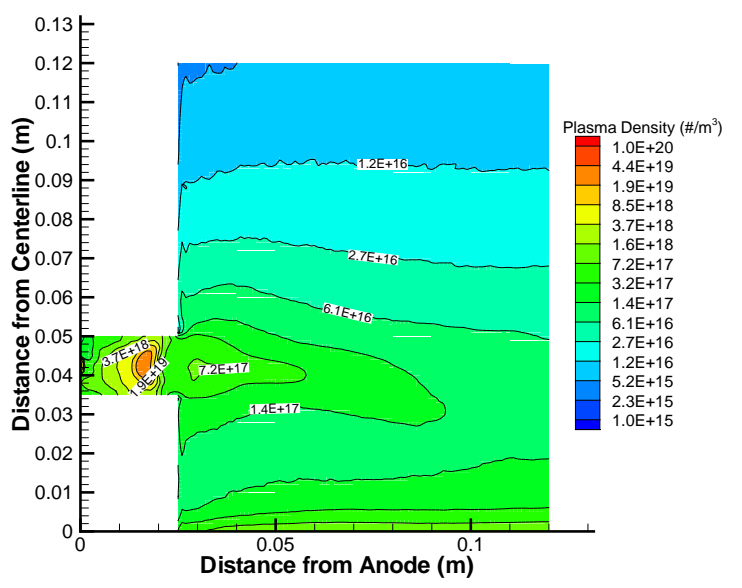

Figure 4. Mean Plasma Density $\lambda=(3)$

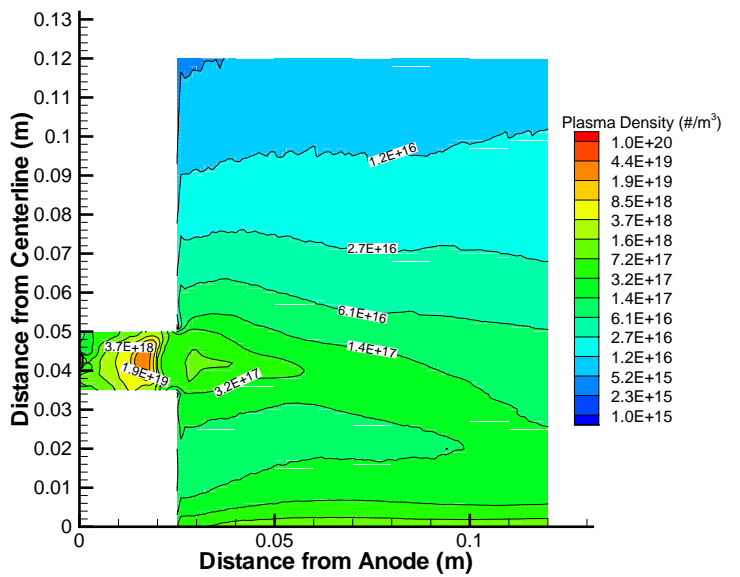

Figure 5. Mean Plasma Density $\lambda=(5)$

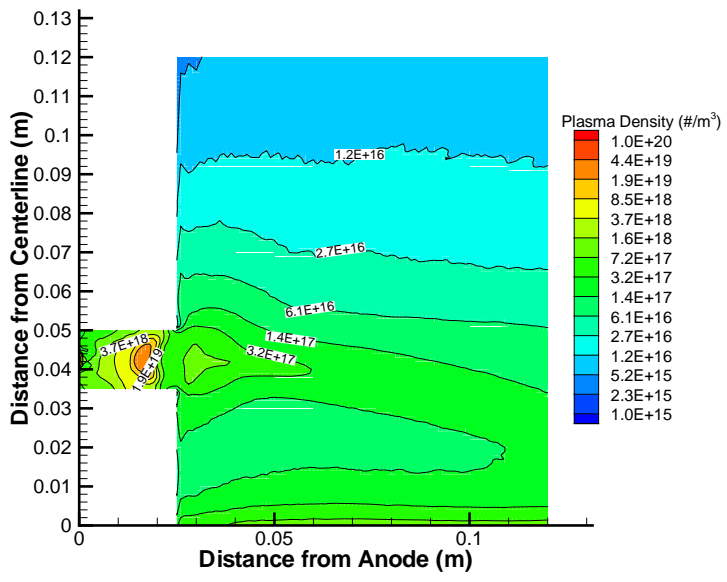

Figure 6. Mean Plasma Density $\lambda=(11)$

\subsubsection{Mean Plasma Potential}

Figures 7-9 show the mean plasma potential for this study. The mean potential profiles for all three simulations are strikingly similar. The potential gradient in the near-field is slightly steeper when the VCL is moved closer to the thruster $[\lambda=(11)]$; however, in all three cases, the location of the steepest part of the potential gradient remains virtually identical. (The nonuniform potential upstream of the virtual anode is due to the fact that the thermalized potential, not the electrostatic potential, is held constant in this region.)

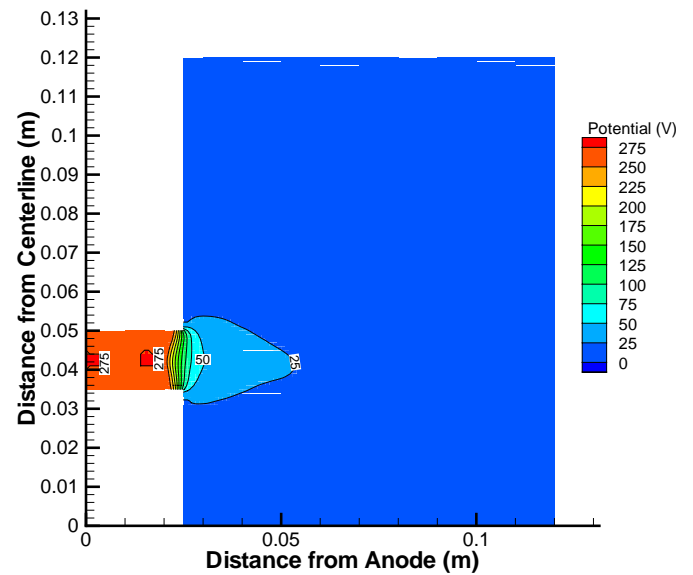

Figure 7. Mean Potential $\lambda=(3)$ 


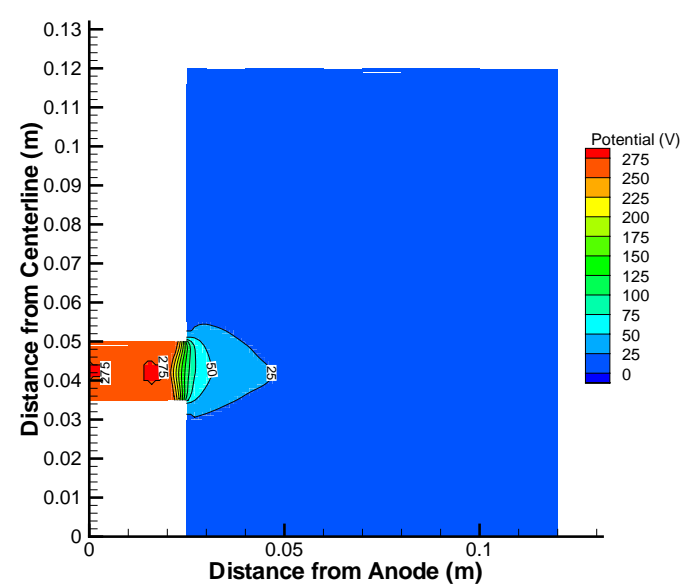

Figure 8. Mean Potential $\lambda=(5)$

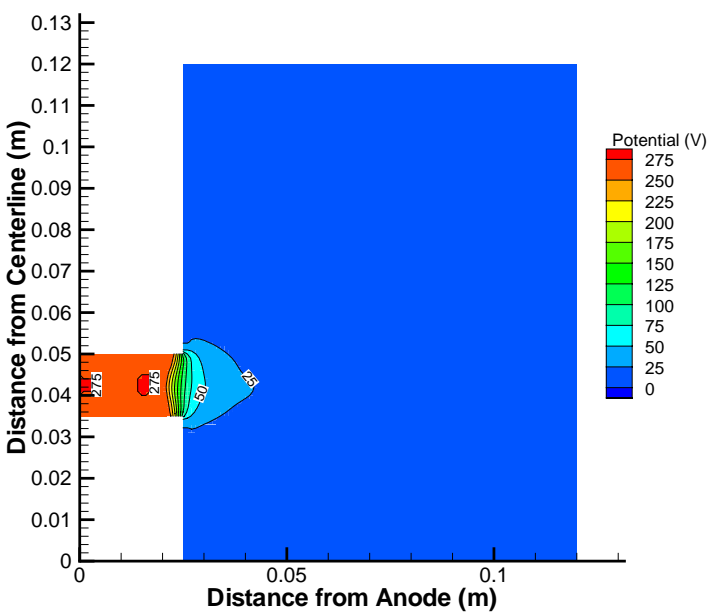

Figure 9. Mean Potential $\lambda=(11)$

3.1.5 Performance Data

\begin{tabular}{|c|c|c|c|}
\cline { 2 - 4 } \multicolumn{1}{c|}{} & Thrust $(\mathrm{mN})$ & Isp (s) & Current (A) \\
\hline Experimental & 80.0 & 1632.7 & \\
\hline$\lambda=(3)$ & 68.9 & 1419.7 & 3.5 \\
\hline$\lambda=(5)$ & 70 & 1464.2 & 3.4 \\
\hline$\lambda=(11)$ & 72.1 & 1510.5 & 3.4 \\
\hline
\end{tabular}

Table 1. SPT-100 Performance Data

The performance results from these results are listed in Table 1 and show that, for this particular configuration, there is little dependence on the location of the VCL. The slightly better performance of the $\lambda=(11)$ case is probably due to less defocusing of the beam in the near field.

\subsubsection{Discussion}

For this SPT-100 type magnetic field configuration, changing the location of the VCL has little effect on the resulting plasma location or potential structure. As a consequence, for all three configurations, the performance parameters calculated by the model are relatively close. Similar results are expected for the P5 magnetic field configuration.

\subsection{Anode Study}

\subsubsection{Motivation}

In previous simulations, assigning the VAL to the injector resulted in increased plasma density near the anode (probably due to the locally elevated neutral density). This increased plasma density alters the potential configuration such that the electron energy climbs and reinforces the high plasma density condition near the anode. The ad hoc solution used in the past for the model is to move the VAL a few centimeters downstream of the injector.

It is believed that the concept of thermalized potential breaks down near the anode due to the dominance of electron diffusion as the primary electron transport mechanism. In addition, the change in magnetic field curvature (aligned with the electric field) near the injector indicates the possibility that the existing 1-D potential solver cannot properly resolve the potential near the anode. For this study, a new anode model is not presented; however, the boundary conditions such a model might provide to the 1-D potential solver are explored through variation of the location of the VAL.

\subsubsection{Configuration}

The location of the VAL is varied for the SPT-100 type magnetic field configuration to study its effect on the performance of this model. Like the VCL, the VAL is typically chosen as a line of constant thermalized potential. For this study, the five VALs chosen correspond to the third through seventh lines of constant thermalized potential from the same magnetic field configuration. The VCL location is chosen as the third line of constant thermalized potential from the domain exit. The five VAL configurations are shown in Fig. 10.

Ionization is permitted upstream of the VAL and the centerline electron energy and thermalized potential are enforced as constants upstream of the VAL. Downstream of the VCL, ionization and wall recombination are permitted and the thermalized potential is held constant. 


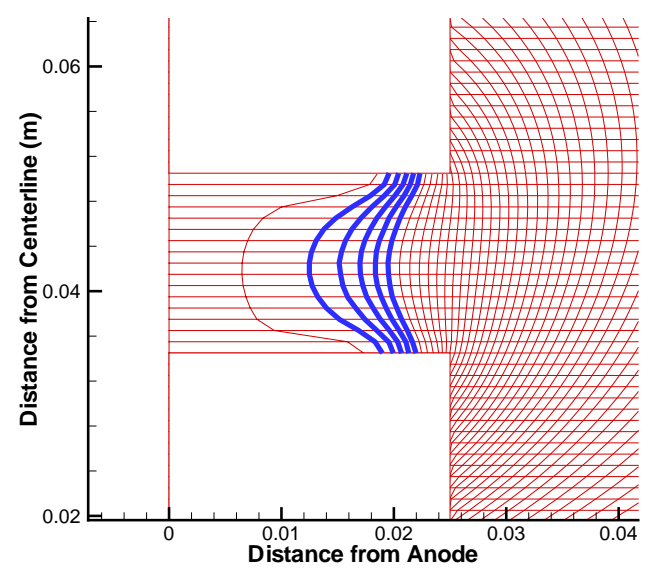

Figure 10. VAL Configurations in bold; (from left to right) $\lambda=3, \lambda=4, \lambda=5, \lambda=6, \lambda=7$

All five models are run with a xenon mass flow rate of $5 \mathrm{mg} / \mathrm{s}$ and imposed potential drop of $275 \mathrm{~V}$ between the VAL and VCL. Doubly charged xenon is not considered in this study.

\subsubsection{Mean Plasma Density}

Unlike the location of the VCL, the location of the VAL has important implications on the resulting mean plasma density, as shown in Figs. 11-15. As the VAL is pushed further and further downstream, the magnitude of the peak plasma density drops substantially. Concurrently, the near-field plasma density also drops substantially. This is most evident in the mean plasma density near the thruster face far off centerline. In addition, the plasma density upstream of the VAL decreases drastically as the VAL is moved downstream, despite the fact that all simulations here employ the same energy boundary condition (corresponding to $\varepsilon=3 \mathrm{eV}$ ) upstream of the VAL. Finally, all cases exhibit a region of high plasma density along the thruster centerline.

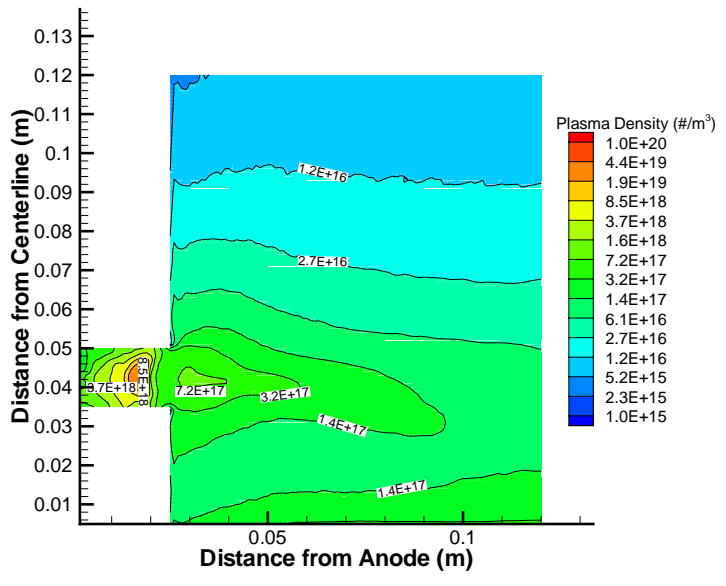

Figure 11. Mean Plasma Density $\lambda=3$

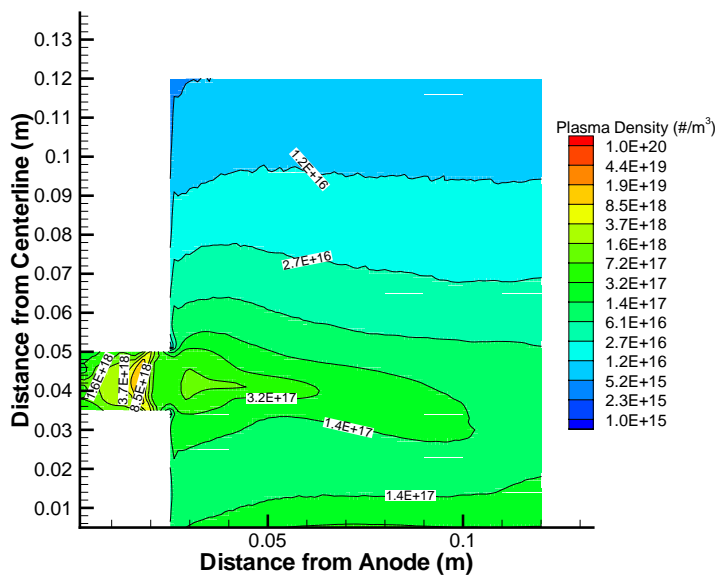

Figure 12. Mean Plasma Density $\lambda=4$

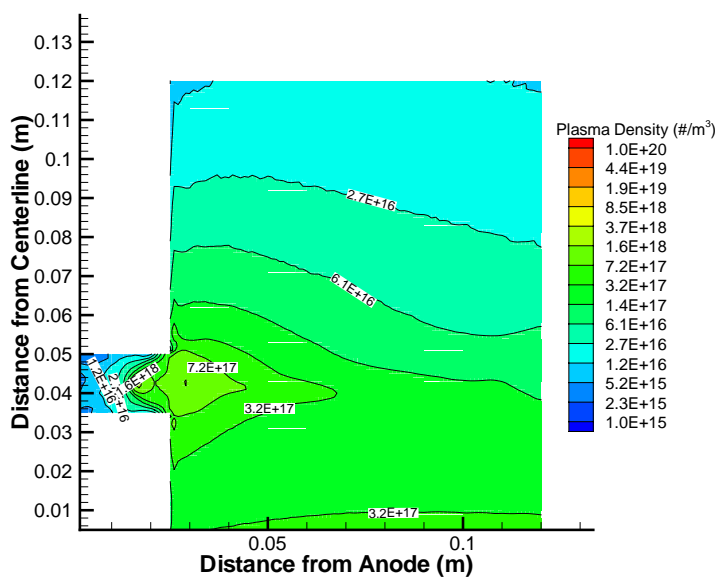

Figure 13. Mean Plasma Density $\lambda=5$ 


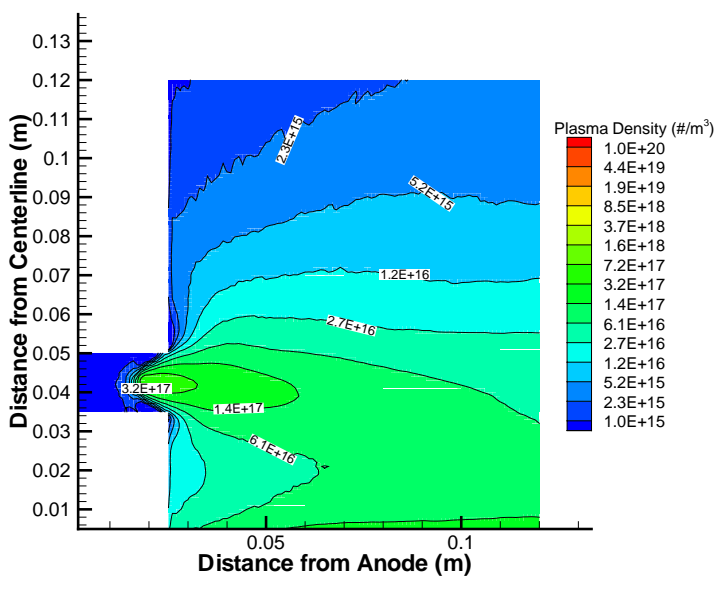

Figure 14. Mean Plasma Density $\lambda=6$

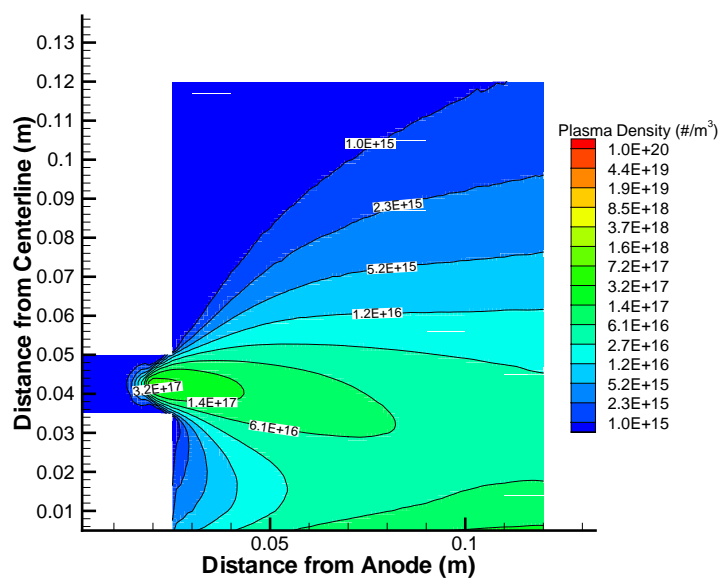

Figure 15. Mean Plasma Density $\lambda=7$

\subsubsection{Mean Neutral Density}

Mean neutral density results for the limiting cases, $\lambda=3$ (near injection VAL) and $\lambda=7$ (near exit plane VAL), are presented in Figs. 16-17. As expected, the higher plasma density of the near injection VAL leads to significant neutral depletion and thus to a much lower mean near-field neutral density relative to the case with the near exit plane VAL; however, the difference in mean neutral density in the region upstream of the VAL is less than an order of magnitude.

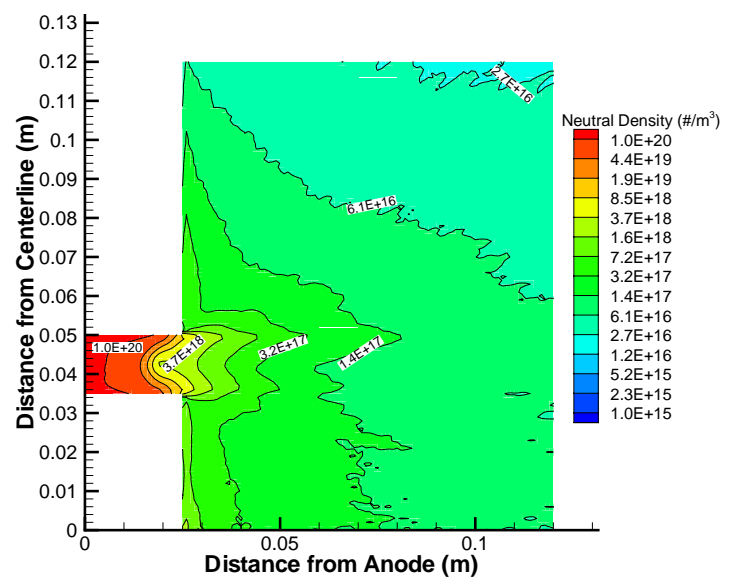

Figure 16. Mean Neutral Density $\lambda=3$

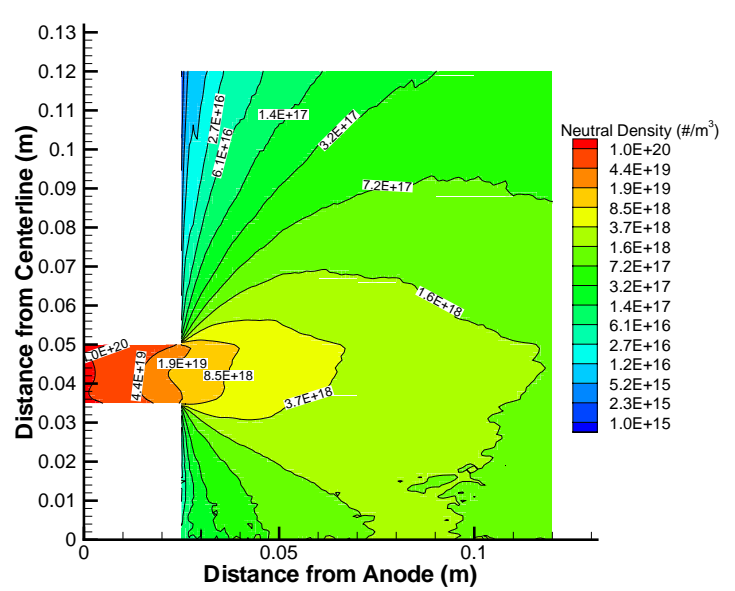

Figure 17. Mean Neutral Density $\lambda=7$

\subsubsection{Mean Electron Energy}

The profiles of mean electron energy for all cases are shown in Fig. 18. It can be noted immediately that for this set of VAL positions, the location of the peak mean electron energy remains largely stationary. For the near injection VAL configuration, the peak electron energy approaches $45 \mathrm{eV}$, while for the near exit plane VAL configuration, the peak electron energy drops to $27 \mathrm{eV}$. This certainly is consistent with the observed peak plasma density magnitudes. For the $\lambda=6$ and $\lambda=7$ cases (where the mean plasma density is lowest), the mean electron energy drops very sharply in the near field while the high mean plasma density cases display an unrealistically high electron energy in the near-field region. 


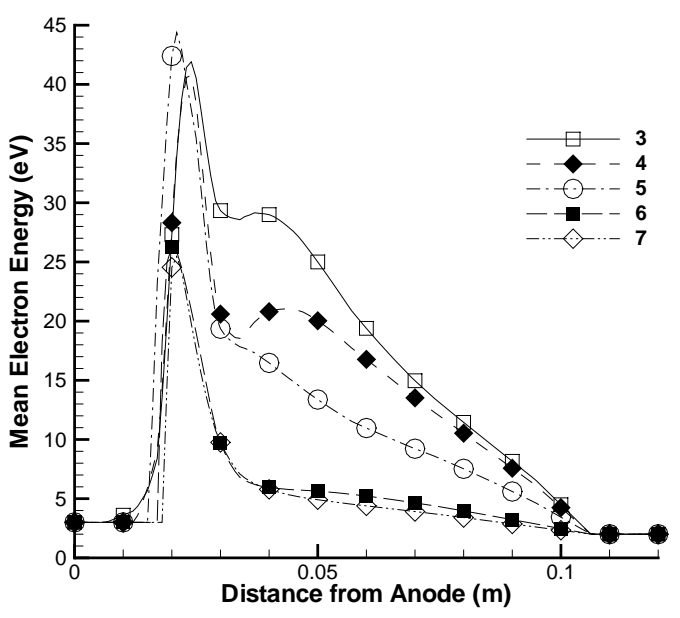

Figure 18. Mean Electron Energy

\subsubsection{Mean Plasma Potential}

Figures 19-20 show that, given the disparities in the mean plasma density between the $\lambda=3$ and $\lambda=7$ cases, the mean potential distributions for these two cases are unexpectedly similar. The largest difference is in the potential decay in the near-field region. For the $\lambda=3$ case, the $25 \mathrm{~V}$ equipotential extends almost $3 \mathrm{~cm}$ into the near-field region while for the $\lambda=7$ case it extends for only $1 \mathrm{~cm}$ into the near-field region.

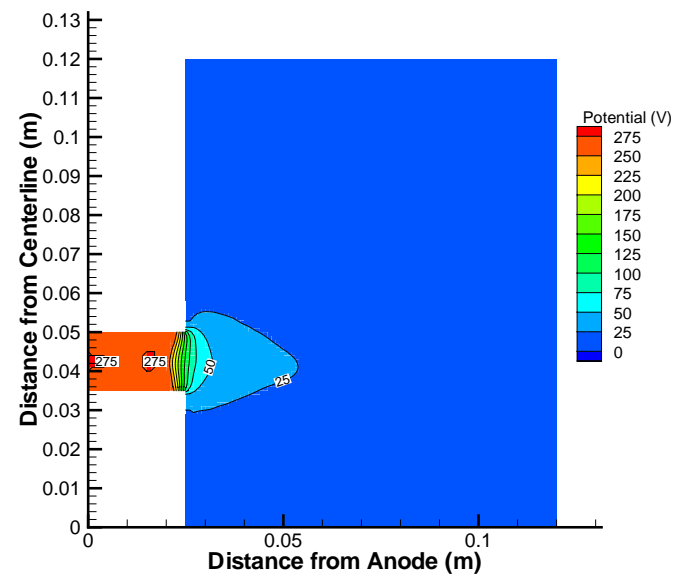

Figure 19. Mean Plasma Potential $\lambda=3$

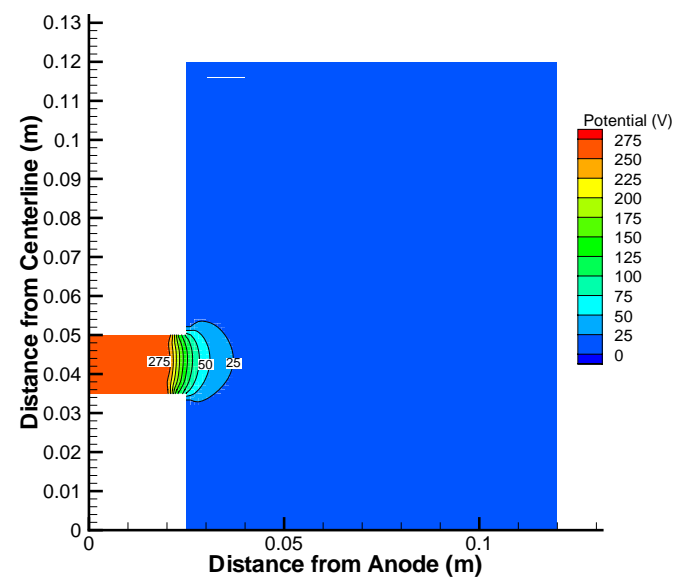

Figure 20. Mean Plasma Potential $\lambda=7$

\section{$\underline{\text { 3.2.7 }}$ Performance Parameters}

\begin{tabular}{|c|c|c|c|}
\cline { 2 - 4 } \multicolumn{1}{c|}{} & Thrust $(\mathrm{mN})$ & Isp (s) & Current (A) \\
\hline Experimental & 80.0 & 1632.7 & \\
\hline$\lambda=3$ & 69.9 & 1425.4 & 3.5 \\
\hline$\lambda=4$ & 66.2 & 1354.1 & 3.4 \\
\hline$\lambda=5$ & 55.8 & 1147.3 & 3.4 \\
\hline$\lambda=6$ & 31.0 & 634.5 & 1.6 \\
\hline$\lambda=7$ & 24.9 & 508.6 & 1.2 \\
\hline
\end{tabular}

Table 2. SPT-100 Performance Parameters

The performance results from this study are listed in Table 2 and show that, for this particular configuration, there is a very strong performance dependence on the location of the VAL.

\subsubsection{Discussion}

As noted, there is significant variation in plasma density upstream of the VAL for the different VAL configurations (over two orders of magnitude). The plasma located in this region must originate either from local ionization or be advected into the region. Since the mean electron energy is constant at $3 \mathrm{eV}$ in this region for all cases, if local ionization is indeed the driving force behind this behavior, then the neutral density should also display a similar two order of magnitude variation. Obviously, based on the mean neutral density results provided in Figs. 16-17, even a single order of magnitude variation in the mean neutral density upstream of the virtual anode does not exist. Thus, it can be concluded, as expected, that the plasma density near the injector is primarily driven by simple ion advection from the source region. 
Elevated near-field mean electron energies are present for the near-injection VAL configurations. For instance, in the $\lambda=3$ case, the location of the $25 \mathrm{~V}$ equipotential (in the near-field region) corresponds roughly to the location of a $25 \mathrm{eV}$ mean electron energy region, while in the $\lambda=7$ case, the same location corresponds to a much lower $10 \mathrm{eV}$ mean electron energy.

One possible explanation for this behavior lies in the differing mean neutral densities in the near-field region. From these results, it appears that in the $\lambda=3$ case, electrons are simply moving up the potential gradient and collecting the full increment of energy from their travel. By contrast, in the $\lambda=7$ case, as the electrons move up the potential gradient, a significant fraction of the energy they gain electrostatically is lost to electronneutral inelastic collisions promoted by the higher neutral density in the near-field region.

Although the position of the VAL has a strong influence on the magnitude of the electron energy distribution (and hence the plasma density) and the shape of the electron energy (especially in the near-field region), the location of peak electron energy remains relatively stationary. Additionally, the potential structure also remains fairly independent of the VAL location. This indicates that a particular magnetic field configuration might contain some fundamentally invariant properties.

Gross performance parameters are typically affected primarily by the magnitude and location of the peak plasma density and the potential structure. Since the location of peak plasma density and the potential structure are largely invariant in this study, trends in performance parameters can be expected to scale with the magnitude of the peak plasma density. Such results are obtained for the configurations tested in this study and are listed in Table 2.

\subsection{Doubly-Charged Xenon}

An experimentally derived P5 magnetic field configuration is tested with both $\mathrm{Xe}^{+}$and $\mathrm{Xe}^{++}$species present. The same configuration is also tested with only a single charged species $\left(\mathrm{Xe}^{+}\right)$present. The magnetic field configuration is provided in Fig. 21.

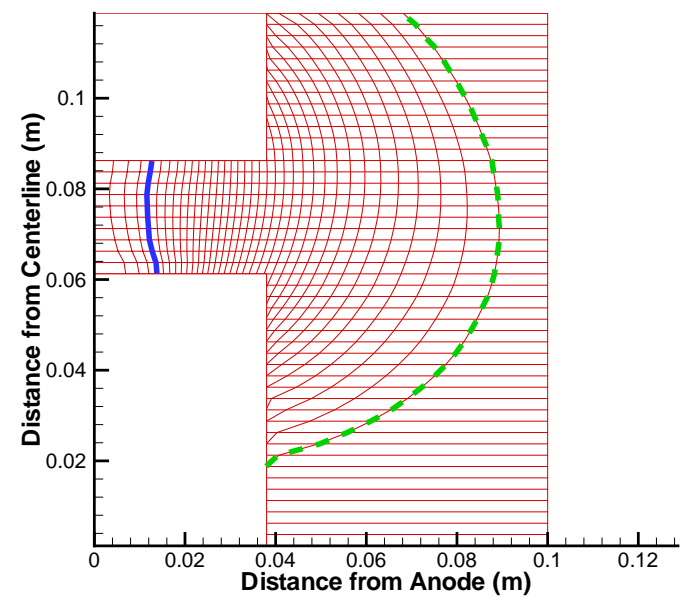

Figure 21. P5 magnetic field configuration; VAL in bold; Dashed line is VCL

Ionization is permitted upstream of the VAL and the centerline electron energy and thermalized potential are enforced as constants upstream of the VAL. Downstream of the VCL, ionization is prohibited but wall recombination can still occur on the outer dielectric walls of the thruster. Heavy particles continue to be tracked to the domain exit boundaries where performance data is evaluated.

Both cases are run with a xenon mass flow rate of 9 $\mathrm{mg} / \mathrm{s}$ and imposed potential drop of $275 \mathrm{~V}$ between the VAL and VCL.

\subsubsection{Mean Plasma Density}

The resulting mean plasma density results for the $\mathrm{Xe}^{+}$ only test are shown in Fig. 22. This unusual doubleplasma peak structure has been observed experimentally for this thruster configuration at low power operation. The relative magnitude of the peak plasma regions in this computational model (with a 1.2 $\mathrm{kW}$ mean power) are both approximately $5 \times 10^{17} 1 / \mathrm{m}^{3}$, which matches relatively well with the $7 \times 10^{17} 1 / \mathrm{m}^{3}$ and $5 \times 10^{17} 1 / \mathrm{m}^{3}$ peaks observed by Haas in a $1.6 \mathrm{~kW}$ experiment. ${ }^{11}$ Comparison with the SPT-100 results shows a much lower peak plasma density, which is characteristic of this thruster, and the aforementioned double peak plasma structure, which is likely due to the particular magnetic field configuration of this thruster. 


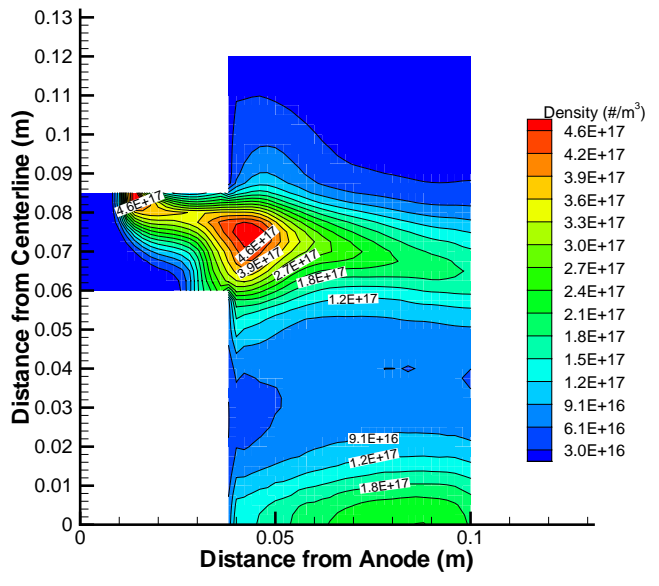

Figure 22. Mean Plasma Density ( $\mathrm{Xe}^{+}$only)

The resulting mean plasma density with the inclusion of $\mathrm{Xe}^{++}$is shown in Fig. 23. The salient feature of this result is a slightly lower mean plasma density near the exit plane of the thruster. This is likely due to the marginal reduction in mean electron energy presented in the next section.

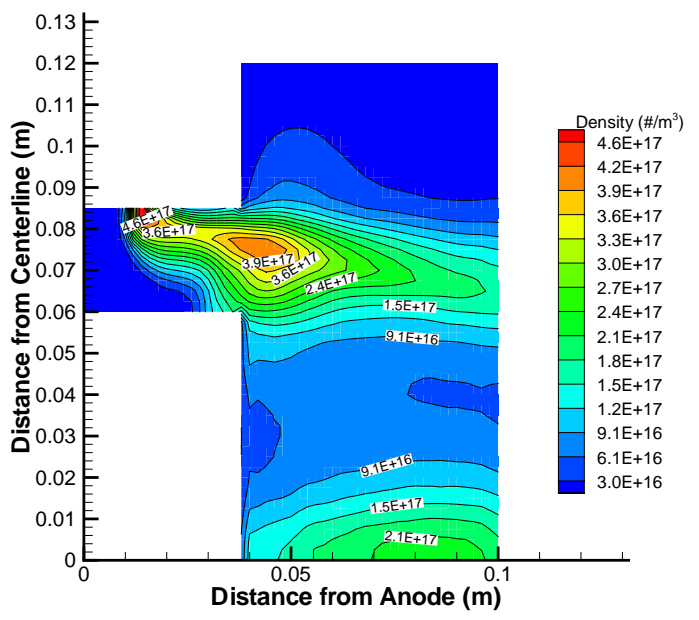

Figure 23. Mean Plasma Density $\left(\mathrm{Xe}^{++}\right.$included)

The resulting $\mathrm{Xe}^{++}$density profiles are shown in Fig. 24. The mean $\mathrm{Xe}^{++}$density profile shows that significant $\mathrm{Xe}^{++}$densities arise solely near the first plasma peak and quickly advect downstream. In magnitude, the $\mathrm{Xe}^{++}$densities approach the $\mathrm{Xe}^{+}$ densities to within an order of magnitude but never exceed that threshold for these simulations.

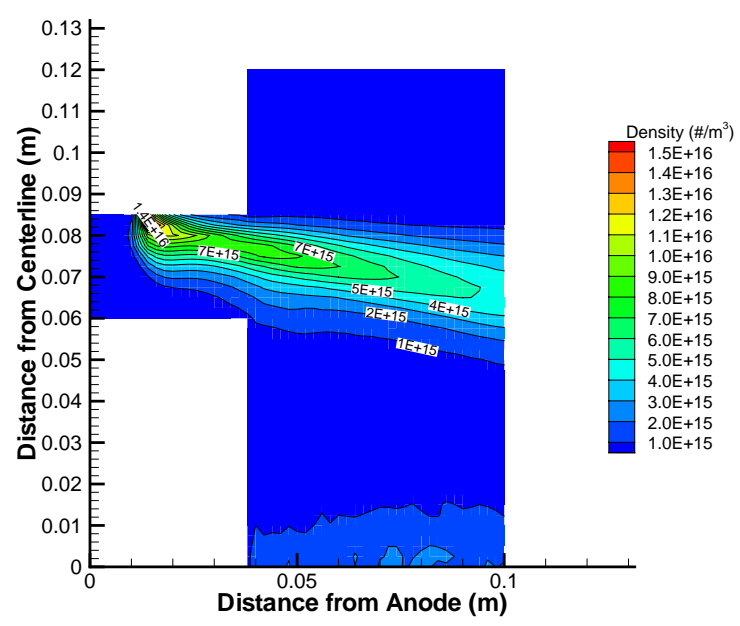

Figure 24. Mean $\mathrm{Xe}^{++}$Density

\subsubsection{Mean Electron Energy}

The mean electron energy associated with both cases is presented in Fig. 25. There is a marginal decrease in peak electron energy due to the additional loss term associated with $\mathrm{Xe}^{++}$ionization. In addition, a small decrease in near field energy is associated with the inclusion of $\mathrm{Xe}^{++}$in the simulation. Nevertheless, both cases displayed a peak electron energy of around $27 \mathrm{eV}$ at a distance of roughly $2 \mathrm{~cm}$ from the anode face. This matches the $27 \mathrm{eV}$ electron energy peak observed by Haas in magnitude; however, the location of the experimental plasma peak of Haas was at $3.5 \mathrm{~cm}$ from the anode face instead of halfway down the acceleration channel. ${ }^{11}$

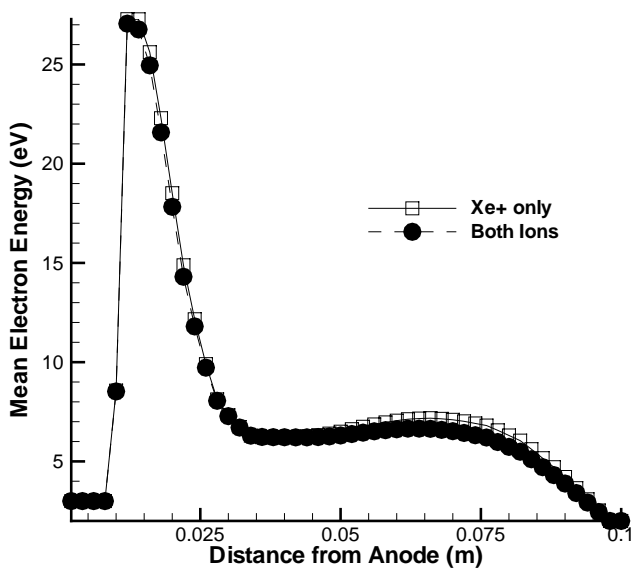

Figure 25. Mean Electron Energy

The shape of the mean electron energy bears a close resemblance to that of the SPT-100 despite the 
significant discrepancies in their mean plasma distributions. In fact, the $\lambda=4$ case of the anode study even displays the same characteristic rise in mean electron energy in the near field region which often occurs in this computational model.

\subsubsection{Mean Plasma Potential}

Based on the relative similarity of the mean plasma density and mean electron energy of the two cases presented here, it is to be expected that the potential structure, which has thus far proved to be relatively insensitive to differences in plasma location, remains unchanged with the inclusion of $\mathrm{Xe}^{++}$. Indeed, the resulting mean plasma potentials from both cases are so nearly identical that Fig. 26 will suffice to illustrate both cases.

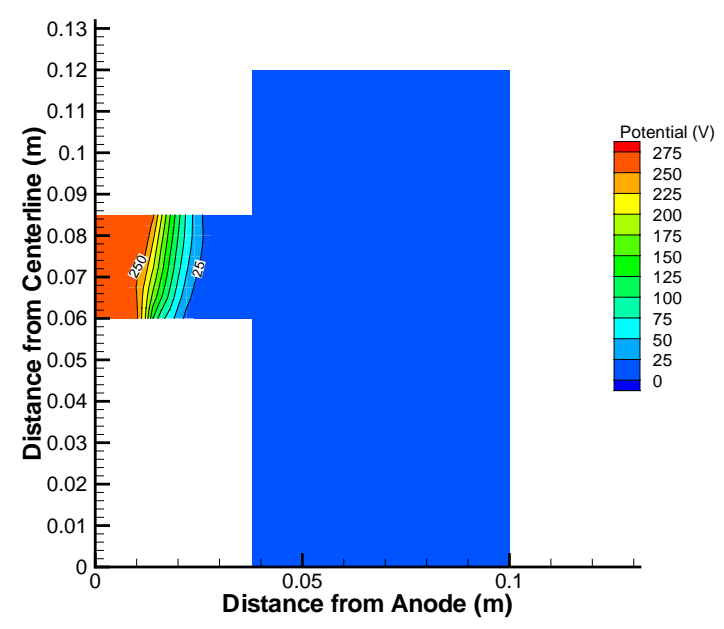

Figure 23. Mean Plasma Potential (both tests)

\subsubsection{Performance Data}

\begin{tabular}{|c|c|c|c|}
\cline { 2 - 4 } \multicolumn{1}{c|}{} & Single & Double & Experimental \\
\hline Thrust $(\mathrm{mN})$ & 83.9 & 86.1 & 95 \\
\hline Isp (s) & 950.4 & 976.3 & 1550 \\
\hline Xe+ Current (A) & 4.3 & 4.1 & \\
\hline Xe++ Current (A) & 0 & 0.3 & \\
\hline Total Current (A) & 4.3 & 4.4 & 5.4 \\
\hline Power (W) & 1185 & 1218.7 & 1600 \\
\hline
\end{tabular}

Table 3. UM/AFRL P5 Performance Data

The model performance data and experimental data, shown in Table 3, are taken at slightly different operating conditions due to time constraints on this paper rather than failings of the model. They are meant to be representative of the performance of this model on the P5 configuration. Not shown is the electron current, which for both P5 cases averaged under $2 \%$ of the total current.

\section{$\underline{3.3 .5 \text { Discussion }}$}

The inclusion of doubly charged xenon into a model configuration based on the P5 magnetic field resulted in a $7 \%$ current fraction of $\mathrm{Xe}^{++}$. Due to the virtually identical potential structure with almost the full potential gradient contained completely within the acceleration channel, only the first region of peak plasma density is expected to contribute significantly to the performance of either the $\mathrm{Xe}^{+}$-only or $\mathrm{Xe}^{+} / \mathrm{Xe}^{++}$ case. Thus, the higher plasma density near the exit plane observed in the singly charged case does not lead to additional performance gains relative to the doubly charged case. On a different magnetic field configuration, such as the SPT-100 type magnetic field, similar behavior might not be expected. Some concern exists over the absence of a significant (10-20\% of total current) electron current in the computational model. Reasons for this discrepancy have not yet been discovered.

\section{$\underline{4.0 \text { Conclusions }}$}

A two-dimensional, unsteady, hybrid PIC-MCC fluid model was applied to an SPT-100 type and a P5 Hall thruster. The boundary condition dependence of a 2-D Hall thruster model based on the assumption of a thermalized potential and a 1-D electron energy equation was studied in this paper. For a given magnetic field configuration, the plasma potential structure seems largely invariant to the location of both the VAL and the VCL. The mean plasma density and mean electron energy showed a strong dependence on the VAL location but not on the VCL location. Moving the VAL downstream resulted in decreased plasma density both in the acceleration channel and upstream of the VAL, reduced thrust, current and Isp, and a lowering of the electron energy in the near-field region. Finally, $\mathrm{Xe}^{++}$was incorporated into the computational model presented in this paper.

\section{$\underline{5.0 \text { Acknowledgements }}$}

The first author gratefully acknowledges financial support from the Department of Energy through a Computational Science Graduate Fellowship and from the University of Michigan through a Rackham Travel Fellowship.

\footnotetext{
${ }^{1}$ Keidar, M., Boyd, I.D., and Beilis, I.I., "Plasma Flow and Plasma-Wall Transition in Hall Thruster Channel," Physics of Plasmas, Vol. 9, 2002, pp. 5315-5322.

${ }^{2}$ Ahedo, E., Martinez-Cerezo, P., and MartinezSanchez, M., "One-Dimensional Model of the Plasma
} 
Flow in a Hall Thruster," Physics of Plasmas, Vol. 8, 2001, pp. 3058-3068.

${ }^{3}$ Roy, S. and Pandey, B.P., "Numerical investigation of a Hall thruster plasma", Physics of Plasmas, Vol. 9, 2002, pp. 4052-4060.

${ }^{4}$ Komurasaki, K. and Arakawa, Y., "Two-Dimensional Numerical Model of a Plasma Flow in a Hall Thruster," Journal of Propulsion and Power, Vol. 11, 1995, pp. 1317-1323.

${ }^{5}$ Fife, J.M., "Hybrid-PIC Modeling and Electrostatic Probe Survey of Hall Thrusters," Doctoral Thesis, Massachusetts Institute of Technology, Department of Aeronautics and Astronautics, September 1998.

${ }^{6}$ Boeuf, J.-P. and Garrigues, L., "Low Frequency Oscillations In a Stationary Plasma Thruster," Journal of Applied Physics, Vol. 84, 1998, pp. 3541-3544.

${ }^{7}$ Hagelaar, G. J. M., Bareilles, J., Garrigues, L., and Boeuf, J.-P., "Two-dimensional model of a stationary plasma thruster," Journal of Applied Physics, Vol. 91, 2002, pp.5592-5598.

${ }^{8}$ Morozov, A. I., Esipchuk, Yu. V., Tilinin, G. N., Trofimov, A. V., Sharov, Yu. A., Shchepkin, G. Ya., "Plasma Accelerator With Closed Electron Drift and Extended Acceleration Zone," Soviet Journal of Plasma Physics, Vol. 17, 1972, p.38.

${ }^{9}$ Garrigues, L., Boyd, I.D., Boeuf, J.P., "Computation of Hall Thruster Performance," Journal of Propulsion and Power, Vol. 17, 2001, pp. 772-779.

${ }^{10}$ Williams, G.J.Jr., Smith, T.B., Gulczinski, F.S., Beal, B.E., Gallimore, A.D., Drake, R.P., "Laser Induced Flourescence Measurement of Ion Velocities in the Plume of a Hall Effect Thruster," AIAA-99-2424, 35 ${ }^{\text {th }}$ Joint Propulsion Conference, Los Angeles, CA, June 20-23, 1999.

11 Haas, J.M, "Low-Perturbation Interrogation of the Internal and Near-Field Plasma Structure of a Hall Thruster using a High-Speed Probe Positioning System," Doctoral Thesis, University of Michigan, Department of Aerospace Engineering, February 2001. 